\title{
Factors Affecting the Measurement of Skin Temperature of Horses Using Digital Infrared Thermography
}

\section{Roy $\mathrm{RC}^{1 *}$, Cockram $\mathrm{M}^{2}$ and Riley $\mathrm{CB}^{3}$}

${ }^{1}$ Large Animal Clinical Sciences, University of Saskatchewan, Saskatoon, SK, Canada

${ }^{2}$ Department of Health Management, University of Prince Edward Island,

Charlottetown, Canadaa

${ }^{3}$ School of Veterinary Science, Massey University, Massey, New Zealand

*Corresponding Author: Roy RC, Large Animal Clinical Sciences, University of

Saskatchewan, Saskatoon, SK, Canada.

DOI: 10.31080/ASVS.2020.02.0085
Received: July 15, 2020

Published: July 27, 2020

(C) All rights are reserved by Roy RC., et al.

\begin{abstract}
Digital infrared thermography has the potential for use as a diagnostic tool to assess the health and welfare of horses in many different ways such as lameness, joint inflammation and pain evaluation. However, many factors affect the surface temperature of horses that are poorly quantified when we use this technology. Unless we quantify these variations and account for these variations while assessments are performed, the reliability of this technology will remain low. In this study, surface temperatures of four different Regions of Interest (head, trunk, front limb and gluteus) of 8 horses were studied in indoor (range $20^{\circ} \mathrm{C}$ to $24^{\circ} \mathrm{C}$ ) and outdoor conditions (range $19^{\circ} \mathrm{C}$ to $22^{\circ} \mathrm{C}$ ). Both the left and right sides of the trunk and gluteal region surface temperatures of horses were measured to determine thermal symmetry. The surface temperature of horses varied significantly depending on the Region of Interest when animals were in outdoor conditions, whereas it remained similar under indoor conditions. Stability of skin temperatures was observed between the left and right side of regions (thermal symmetry) even under outdoor conditions. Moreover, digital infrared thermography performed in a controlled environment provides more reliable estimates of surface temperature. If animals are exposed to the outdoor environment before thermography, adequate equilibration time should be provided for the surface temperature to revert to normal surface temperatures.
\end{abstract}

Keywords: Horse; Infrared; Thermography; Skin Surface; Temperature

\section{Abbreviations}

DT: Digital Infrared Thermography; ROI- Region of Interest

\section{Introduction}

Skin temperature, as measured by digital infrared thermography (DT), can be used effectively for diagnostic purposes, when all the factors which affect the skin temperature measurements are quantified. Energy is emitted through the skin of an animal, and when measured as skin temperature varies depending upon surface temperature, surrounding environmental factors, and the surface characteristics of the object being imaged [1]. Outdoor fac- tors such as sunlight, humidity, ambient temperature [2] and wind speed [3] may also have an effect on skin temperature measured. The technique used by the operator affects outcomes including distance from the target, viewing angle and motion. Factors associated with the animal itself and its individual response to the surrounding environment may also influence the qualitative or quantitative data generated by DT. These include the level of activity prior to assessment [4], the physiological state of the animal (e.g. pregnancy) [5], the length and density of the hair coat [6], any concurrent pain or stress $[7,8]$ and disease status [9]. Endogenous animal factors may be difficult to compensate for in DT data analysis, but it is hy- 
pothesized that the techniques can be standardized if environmental factors are understood and accounted for in the interpretation of results.

In human medicine, thermography is often used as a diagnostic tool under controlled environmental conditions [10]. However, in veterinary medicine, it is often difficult to control the environment in which thermographic images are taken [4,9]. Some of the published work reporting on the use of DT with horses under outdoor conditions suggests that it may be used successfully in such circumstances. One group of researchers used DT on horses to examine cold tolerance in different seasons in a riding arena [5]. However, in a subsequent study, problems were reported in accurately determining heat loss patterns with DT at very cold temperatures $\left(-21^{\circ} \mathrm{C}\right)$, particularly when snow was present on the animal's body surface [10]. Another study that examined the reliability and repeatability of skin temperatures measured by DT found that the skin temperature patterns in horses were reproducible for up to seven days when environmental conditions were similar, suggesting that there may not be a need for an equilibration time [6].

The development of DT as a semi-quantitative tool has the potential as a reliable tool to detect stress, pain and subclinical or non-visible injuries. To achieve this goal, understanding the operator and animal-related factors that affect skin temperature measured by DT under different environmental conditions is important for its use in the evaluation of animal welfare in field conditions.

\section{Objective of the Study}

The objective of this study was to examine in clinically normal horses at rest, and evaluate the effects of the following on skin temperatures as measured by DT:

- Variation of the distance from which reliable thermographic images may be expected to be obtained under field conditions;

- Variations in skin temperatures among anatomic regions of interest (ROIs);

- $\quad$ Right versus left-sided thermal symmetry; and

- Environmental conditions (indoor versus outdoor).

\section{Materials and Methods}

Animals

Eight clinically healthy Standardbred horses (five mares and three geldings) between 5 and 22 years of age (median 11 years) were used following the approval of the Animal Care Committee at the University of Prince Edward Island (UPEI).

\section{DT image acquisition}

Digital infrared thermography data was obtained for each horse in standardized indoor and outdoor locations at UPEI with an infrared thermographic camera (ThermaCAM SC 2000 thermographic camera, FLIR system Inc., Wilsonville, OR 97070). All indoor images were taken during a single week when the animals were at rest and normally housed indoors in the Atlantic Veterinary College premises (temperature range 20 to $24^{\circ} \mathrm{C}$; humidity range 20 to $58 \%$ ). All outdoor images were obtained when the same animals were at rest in an immediately adjacent small paddock measuring $7 \mathrm{~m}^{2}$ (temperature range 19 to $22^{\circ} \mathrm{C}$; humidity range 40 to $53 \%$ ) on a different week (four weeks apart). The environmental temperature, relative humidity - measured using a hygrometer (Magna-Temp RH\%, Canadian Tire, Toronto, ON) and distance between camera and the horse were measured using a laser distance meter (Master $\mathrm{craft}^{\circledR}$ Hawk eye laser, Model no: LM04CN, Canadian Tire, Toronto, ON) during the capture of each DT image. The DT image with the correlated temperature data map generated by the camera hardware were saved and downloaded on to a computer equipped with software for image evaluation (ThermaCAM Researcher Professional 2.8 SR-3 software, FLIR system Inc., Wilsonville, OR 97070). Skin temperature readings were adjusted using this software for an object parameter emissivity value of 0.95 [7], distance between the horse and camera, environmental temperature and relative humidity.

To evaluate the effect of the distance from the horse (an operator factor) on DT based skin temperature measurements, thermographic images were obtained for each horse under indoor and outdoor conditions for the following ROI's: anterior view of the head region at 4 and 5 metres; left lateral view of the trunk region at 5 and 6 metres; right lateral view of the trunk region at 5 and 6 metres; left lateral view of the left front limb (carpus to hoof) at 1 and 2 metres, and; posterior view of the buttock region at a distance of 4 metres. Distances were chosen based on the field of vision attainable using the DT camera, and on expected field conditions anticipated at slaughter plants processing horses where close proximity to the horse is usually not be possible.

\section{Data and statistical analysis}

Mean skin temperature was calculated for each image from DT data points over a straight line drawn between two anatomic 
points, for each anatomic region. The thermography software was used to calculate the mean skin temperature by selecting sample temperature at several points (depending on the image pixel density) along the anatomical line chosen. The mean temperature of each head image was obtained over a line drawn from the mid-eye level to the middle of the bridge of the nose between the external nares (Figure 1a and 1b). The mean temperature of each trunk image was obtained over a line drawn from the highest point of the withers to the lowest point of the flank (Figure 2a and 2b). The mean front limb temperature was measured over a line drawn from the mid-carpus level to the mid-fetlock joint (Figure 3a and 3b).The mean temperature of each image of the gluteal/caudal thigh region was obtained over a line drawn between the level of tuber ischium (buttock) downwards to the lower thigh (where the semimembranosus and gracilus muscles intersect) (Figure 4).



1a: DT image at a distance of five metres.

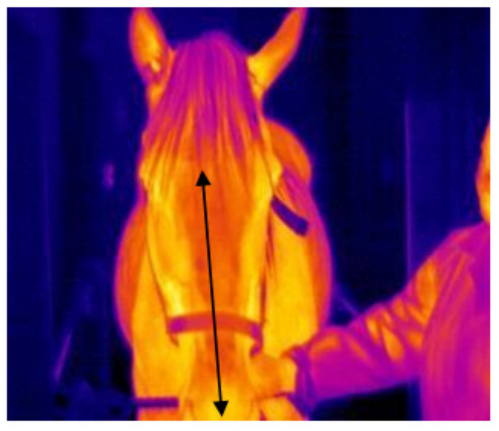

1b: DT image at a distance of four metres.

Figure 1a and 1b: Digital infrared thermographic images of the frontal head region at four and five metres, and the line between selected anatomical landmarks (mid-eye level to mid-point between the external nares) over which mean skin temperatures were calculated.

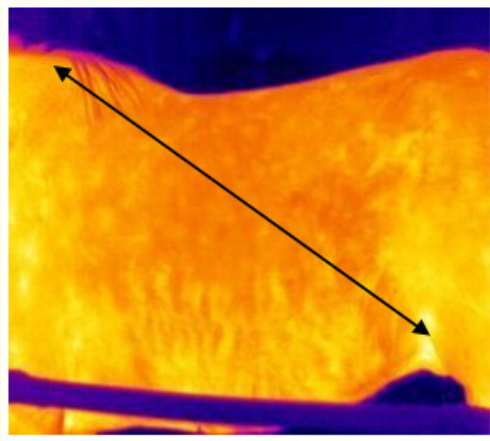

2a: DT image at a distance of six metres.

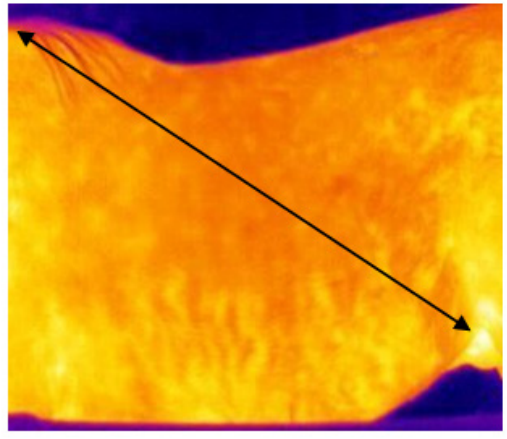

2b: DT image at a distance of five metres.

Figure 2a and 2b: Digital infrared thermographic images of the lateral trunk of a horse taken from a distance of five and six metres. The line shows the span between selected anatomical landmarks (highest point of withers to the lowest point of the flank) over which mean temperatures were calculated. 


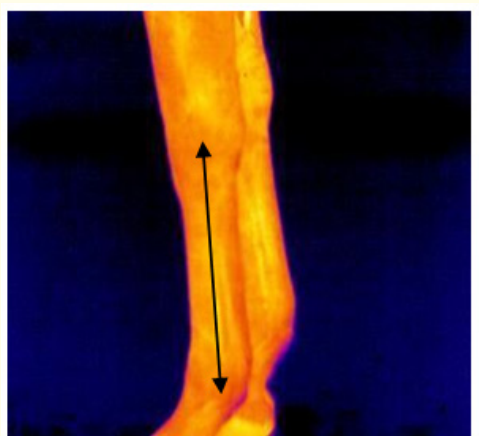

3a: DT image at a distance of two metres.

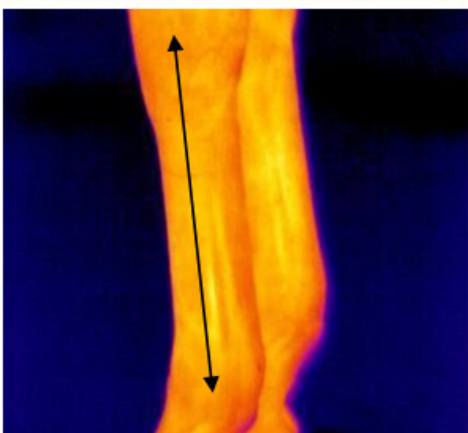

3b: DT image at a distance of one metre.

Figure 3a and 3b: Digital infrared thermographic images of the left lateral limb of a horse taken from a distance of one and two metres. The line drawn between two anatomical landmarks (mid carpus and fetlock joint) shows the landmarks over which mean temperatures were calculated.

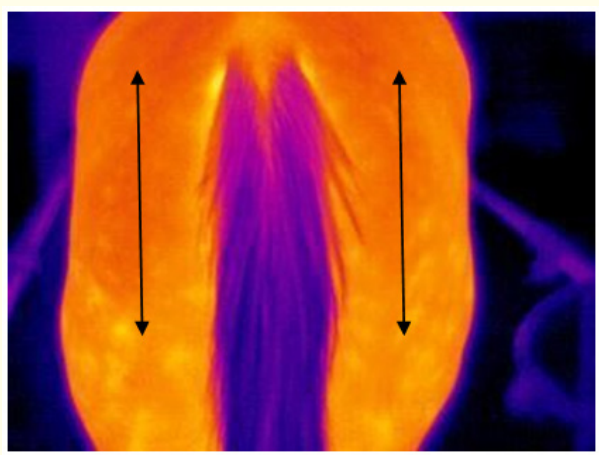

Figure 4: Digital infrared thermographic image of the posterior gluteal/caudal thigh region of a horse taken from a distance of four metres. The line drawn between the two anatomical landmarks (level of tuber ischium to the level where the semimembranosus and gracilus muscles intersect (lower thigh) shows the landmarks over which mean temperatures were calculated.

The mean skin temperatures measured under different conditions were calculated. The effects of the camera distance from the horse, environmental conditions (indoor and outdoor), left or right sides (symmetry) and ROI were first evaluated unconditionally, and then modelled together in a multiple regression model to understand the interactive effects of each variable on the mean skin temperature measured. To account for repeated measures, a mixed model regression analysis approach was employed, with the animal (subject) as a random effect [8]. Nonsignificant effects ( $\mathrm{P} \geq$ 0.05 ) were dropped in the final model and for the others parameter estimates and predicted medians were obtained. To meet model assumptions (residuals to be normally distributed), the outcome (skin temperature) was log-transformed after subtraction of an offset value $\left(23^{\circ} \mathrm{C}\right)$ determined by a Box-Cox type analysis [9]. Residuals of the model were checked for normal distribution and heteroskedasticity using graphical methods. Post estimation pairwise comparison of temperatures was performed between indoor and outdoor conditions for each ROI. The significance was set at $\mathrm{P}<$ 0.05 . Statistical analyses were carried out using the software (Stata 12.1, StataCorp, 4905 Lakeway Drive College Station, Texas 77845, USA.), except for the Box-Cox analysis which utilized the MASS library for R software (R Foundation, Vienna, http://www.r-project. org/).

\section{Results and Discussion}

The median and interquartile range of skin temperatures with respect to distance, ROI, symmetry and environmental conditions are listed in table 1. Descriptive statistical mean skin temperatures calculated and statistical estimates from the model for ROI's under outdoor conditions were highest in the trunk region, followed by gluteal/caudal thigh regions, limb and frontal head regions as illustrated in table 1 and figure 5 respectively. The ROI's selected and environmental conditions significantly affected mean skin temperatures as measured by DT (Table 2). Skin temperatures under outdoor conditions were significantly different among the ROI's examined except between the head and limb regions. However, there were no significant differences in the skin temperatures among different ROI's under indoor conditions. 


\begin{tabular}{|c|c|c|c|c|}
\hline \multirow{2}{*}{ Factors } & \multirow{2}{*}{ ROI } & \multirow{2}{*}{$\mathbf{n}^{*}$} & \multicolumn{2}{|c|}{ Median skin temperature (interquartile range) ${ }^{\circ} \mathrm{C}$} \\
\hline & & & Indoor & Outdoor \\
\hline \multicolumn{5}{|l|}{ Distance (m) } \\
\hline 1 & Limb & 8 & $32.3(30.1-32.9)$ & $32.5(31.2-34.8)$ \\
\hline 2 & Limb & 8 & $32.4(29.6-32.9)$ & $31.8(29.9-34.6)$ \\
\hline 4 & Head & 8 & $31.8(30.1-32.9)$ & $31.5(31.2-32.4)$ \\
\hline 5 & Head & 8 & $31.9(30.6-32.8)$ & $31.9(31.0-32.3)$ \\
\hline 4 & Gluteus & 16 & $32.6(30.4-33.3)$ & $35.3(34.3-36.8)$ \\
\hline 5 & Trunk & 16 & $31.7(30.3-31.7)$ & $36.3(35.4-41.4)$ \\
\hline 6 & Trunk & 16 & $31.9(30.2-32.6)$ & $36.3(34.7-40.9)$ \\
\hline \multicolumn{5}{|l|}{ Symmetry } \\
\hline Left & Trunk & 16 & $31.7(30.2-32.6)$ & $35.8(34.9-39.8)$ \\
\hline Right & Trunk & 16 & 31.7 (30.1-32.7) & $37.4(35.1-41.1)$ \\
\hline Left & Gluteus & 8 & $32.6(30.3-33.0)$ & $35.3(34.3-36.4)$ \\
\hline Right & Gluteus & 8 & $32.7(31.7-33.4)$ & $35.6(33.9-36.9)$ \\
\hline
\end{tabular}

Table 1: The median and interquartile ranges of skin temperatures measured at four regions of interest (ROI) on eight resting healthy horses by digital infrared thermography tabulated by distance, region of interest (ROI), symmetry and environmental conditions.

$\mathrm{n}^{*}=$ no. of DI images, $\mathrm{m}=$ metre $/ \mathrm{s}, \mathrm{C}=$ Celsius.

\begin{tabular}{|l|c|c|c|c|}
\hline \multirow{2}{*}{ Predictor } & \multirow{2}{*}{ Levels } & \multicolumn{3}{|c|}{ Parameter estimates (log) } \\
\cline { 2 - 5 } & Head & $\mathbf{( - \mathbf { C } )}$ & $\mathbf{S E}$ & P-Value \\
\hline ROI -Indoor & Trunk & ref & $\mathrm{n} / \mathrm{a}$ & $\mathrm{n} / \mathrm{a}$ \\
\hline & Gluteal/caudal thigh & -0.01 & 0.05 & $\mathrm{~ns}$ \\
\hline \multirow{2}{*}{ Environment } & Limb & 0.07 & 0.06 & $\mathrm{~ns}$ \\
\hline & Indoor environment & ref & $\mathrm{n} / \mathrm{a}$ & $\mathrm{n} / \mathrm{a}$ \\
\hline \multirow{2}{*}{ ROI } & Outdoor environment & 0.04 & 0.06 & $\mathrm{~ns}$ \\
\hline $\boldsymbol{x}$ Environment & Head $\boldsymbol{x}$ outside & ref & $\mathrm{n} / \mathrm{a}$ & $\mathrm{n} / \mathrm{a}$ \\
& Trunk $\boldsymbol{x}$ outside & 0.52 & 0.07 & $<0.001 \dagger$ \\
& Gluteal $\boldsymbol{x}$ outside & 0.31 & 0.08 & $<0.001 \dagger$ \\
\hline Constant & Limb $\boldsymbol{x}$ outside & 0.09 & 0.08 & $\mathrm{~ns}$ \\
\hline
\end{tabular}

Table 2: The parameter estimates with standard errors of the coefficients (SE) and the P values for the mixed model regression analysis between log-transformed skin temperature measurements using DT and predictor: region of interest (ROI) and environment. The overall model significance by Wald test was $\mathrm{P}<0.001$.

ref = Reference level in the model; $\mathrm{ns}=$ Not significant; $\boldsymbol{x}=$ Interaction term; $†$ Significant interaction at the level of $\mathrm{P}<0.05 ; \mathrm{n} / \mathrm{a}=\mathrm{Not}$ applicable. 


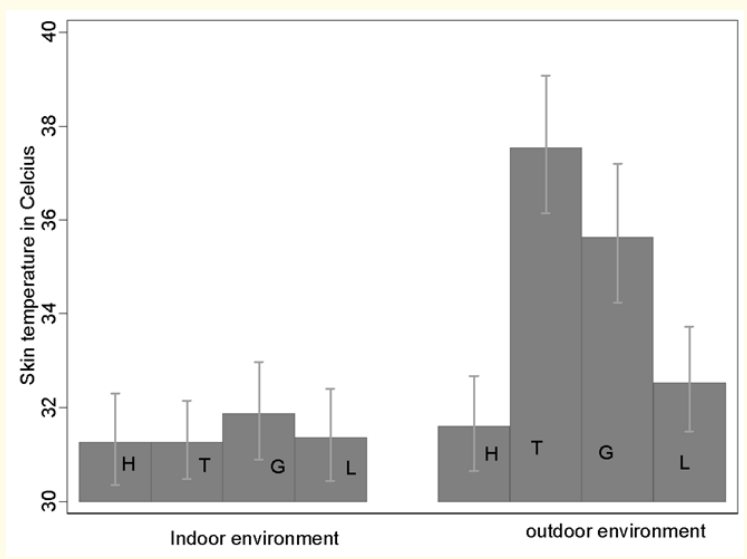

Figure 5: The model based predicted median and confidence interval of temperatures for each region ( $\mathrm{H}=$ head, $\mathrm{T}=$ trunk, $\mathrm{G}=$ gluteal/caudal thigh, $\mathrm{L}=$ limb) under indoor and outdoor environments. The estimates were back-transformed to the original scale $(\stackrel{\circ}{\circ})$ from the modelled data summarized in table 2 .

The distance at which DT imaging was performed did not have a significant effect on skin temperatures as measured by DT at the ranges compared. Thermal symmetry (left or right side of the body) did not have any significant effect on the skin temperatures calculated using DT. There were significant interactions between ROI's and whether thermography was performed indoors or outdoors. Pairwise comparisons of each ROI's (4 groups) between indoor and outdoor environments (2 groups) showed that all regions except the head had significantly higher temperatures when DT was performed under outside conditions (Figure 5).

Previous studies of DT in cattle and dogs have indicated that different ROIs of the same animal can have varying skin temperatures as measured by DT $[2,10]$. In the current study under indoor conditions, the posterior gluteal/caudal thigh region had an elevated mean skin temperature compared with other regions, even though this was not statistically significantly different (Table 1). This elevated mean skin temperature was not unexpected. There are significant variations in skin thickness and subcutaneous vasculature among regions, resulting in areas where the outer skin surface is in close proximity to blood vessels, producing higher mean skin temperatures [11]. Different parts of the body may also respond differently to the sympathetic nervous system stimulation, resulting in varying amounts of vasoconstriction and vasodilatation, subsequently affecting local blood flow, and thereby the DT determined skin temperatures of ROIs [10]. However, in this study, the lack of significant differences in skin temperature among three regions (head, trunk and limbs) in the indoor environment indicated that the interaction with the environment may be a more critical determinant of skin temperature variability [2].

The lack of significant regional skin temperature differences in normal horses using DT under indoor conditions has not, to the authors' knowledge, been reported previously. However, a similar study in healthy humans under indoor conditions resulted in similar findings among mean skin temperatures for the trunk and cheek regions [12]. However, the extremities of human body (the feet) had different skin temperatures that were attributed to differences in circulation and tissue metabolism [12]. Recording of stable mean skin temperatures in different ROIs of healthy horses using DT under indoor conditions in this study supports the use of this tool for evaluation of pathologies that may increase surface temperature, such as infection, inflammation, pain and stress $[13,14]$.

The use of DT in an outdoor environment affected skin temperatures measured by DT in this study. This supports the findings of others, where heat loss from different regions, for example the neck region versus the trunk region, has been shown to differ significantly in outdoor conditions [5,15]. Higher temperatures in the trunk region are reflective of greater rates of heat loss under outdoor conditions [5]. Although the horses in the current study were stationary when evaluated by DT, it should also be noted that muscular activity may be greater when animals have the opportunity to move around, resulting in greater heat production by muscle as a by product of increased metabolic activity [16]. Increased heat production in muscles increases the body's core body temperature. In this situation, heat generated in the muscles may be transported to skin by blood [17], thereby increasing DT temperature readings. For this reason, the authors suggest that optimally, horses should be provided with a period of rest or equilibration prior to assessment in outdoor environments [18].

Another plausible factor that has been suggested to account for regional differences in skin temperature is exposure to direct sunlight [19] in outdoor environments. However, under the temperate environmental conditions of the study, there was no evidence of thermal asymmetry that may have otherwise supported a role for this factor in the study environment. This finding was in agreement with the work of others [20] where the left and right side buttock skin temperature measurements were not significantly different. 
This is an important finding with respect to finding pathologies in different body parts of the animal, where one side may usefully provide the basis of comparison for the other side.

The ambient temperature has been known to affect skin temperatures measured under outdoor conditions in cattle and horses $[2,5,15]$. However, in one study, no changes in skin temperature were recorded when the air temperature dropped by $3.1^{\circ} \mathrm{C}$, but a significant response was found when the air temperature dropped by $6.5^{\circ} \mathrm{C}[21]$. In the current study, the difference between the ambient temperature of the indoor and outdoor conditions was minimal $\left(<3^{\circ} \mathrm{C}\right)$. The outdoor and indoor temperature range during this study was within the thermo-neutral zone of horses unaccustomed to winter weather $\left(5-25^{\circ} \mathrm{C}\right)$ where a minimal change in metabolic rate would be anticipated due to ambient temperature [22].

Mean skin temperatures measured using DT at different distances of different regions ( $1 \mathrm{~m}, 2 \mathrm{~m}, 4 \mathrm{~m}, 5 \mathrm{~m}$ and $6 \mathrm{~m})$ did not differ significantly. The distances evaluated were chosen for practical considerations with the view that these reflected the variability that might be experienced while attempting to utilize DT under field conditions encountered (e.g. in slaughter plants). Such conditions may include an auction yard sale, slaughter plant or large animal diagnostic situation. The power of infrared radiation is independent of the distance $\left(P=\sum \int \mathrm{A}\left(\mathrm{T}^{4}-\mathrm{T}_{\mathrm{c}}^{4}\right)\right.$, where $P=$ net radiated (infrared) power, $\Sigma$ = emissivity; $\Upsilon=$ Stefan-Boltzmann constant $5.6703 \times 10^{-8} \mathrm{~W} / \mathrm{m}^{2} \mathrm{~K}^{4} ; \mathrm{A}=$ radiating area; $\mathrm{T}^{4}=$ temperature of radiator; $\mathrm{T}_{\mathrm{c}}{ }^{4}=$ temperature of surroundings), but attenuation may occur as a result of atmospheric absorption of radiation, particularly in situations of high humidity [23]. In the current study, the degree of humidity appeared to have minimal impact on the data, and the variation in distances was apparently insufficient for the thermographic camera to record a significant difference in temperature. Larger discrepancies may occur with larger differences in distances from the target of interest, due to the finite pixel density of the camera's infrared sensor, and changes in the relative area being assessed with respect to the Stefan-Boltzman equation. In such circumstances, each pixel represents the average temperature of a greater focal area of the target as the distance between camera and horse increases. The use of the camera at close quarters may be associated with a reduction in the impact of outliers by measuring the skin temperature of a larger zone rather than a spot temperature measurement. However, under field conditions, proximity to the horse may increase the risk of injury to the operator, and stress on the horses due to the approach of unfamiliar personnel.
The thermal camera and proprietary software used has inbuilt provisions to adjust the skin temperature measured according to the distance from which the images are taken, and it appears that the experimental conditions of the current study did not exceed this capacity.

In this study, mean skin temperature of a vector across the ROIs was utilized rather than spot skin temperature measures. Recording mean skin temperature of a larger area of a particular region may provide more useful information than spot temperature, particularly when DT is used for the detection of bruising ante-mortem in livestock before slaughter. For example, a single spot measure of temperature may miss a smaller lesion in a particular ROI, whereas the mean skin temperature of the whole ROI may detect the lesion with more sensitivity. However, the drawback in using mean skin temperature is that any outlier values for temperature would be less apparent as they will be averaged out. This drawback may be mitigated by using maximum and minimum skin temperature values, standard deviation or confidence interval of skin temperature.

One practical challenge experienced during this study related to usage of DT was the inconvenience of manually recording environmental temperature, humidity and distance during the process of obtaining each DT capture. The authors suggest that the incorporation of an inbuilt distance measuring tool, environmental temperature and humidity measuring capabilities would help operators to detect thermal changes with significant ease. This would enhance the handling of DT and make it a simpler and more user friendly diagnostic tool under outdoor conditions.

\section{Conclusion}

In conclusion, within the conditions of the study, the reliability of DT based skin temperature measurements was better under controlled indoor conditions than under outdoor conditions. To use DT to detect injuries under outdoor conditions more controlled studies at a greater range of ambient temperatures should be conducted. Significant differences in skin temperature among ROI's was noticed when DT was performed under outdoor conditions. However, the stability of skin temperatures was observed between left and right side of regions (thermal symmetry) even under outdoor conditions. This stability is useful when attempting to detect asymmetric thermal indicators of pathology. The differences in DT results between indoor and outdoor conditions of the study were not unexpected. This work sought to develop a means to quantitatively assess them under the conditions of the study, and to identify 
where these differences might most critically impact on objective thermographic detection of abnormalities in the future application of this modality.

\section{Acknowledgement}

I am grateful to the physics department of the University of Prince Edward Island for use of the research grade infra-red thermography camera. This is part of my $\mathrm{PhD}$ research. I am grateful to Dr. Michael Cockram and Dr. Chris Riley for helping me design, perform and write this manuscript.

\section{Conflict of Interest}

No conflict of interest.

\section{Bibliography}

1. Holst GC. "Common sense approach to thermal imaging". JCD Publishing and SPIE The International Society for Optical Engineering, United States of America (2000).

2. Alsaaod M and Büscher W. "Detection of hoof lesions using digital infrared thermography in dairy cows". Journal of Dairy Science 95 (2012): 735-742.

3. Westerman S., et al. "The effect of airflow on thermographically determined temperature of the distal forelimb of the horse". Equine Veterinary Journal 45 (2013): 637-641.

4. Gloster J., et al. "Normal variation in thermal radiated temperature in cattle: implications for footand-mouth disease detection". BMC Veterinary Research 7 (2011): 73-82.

5. Autio E., et al. "Measuring the heat loss in horses in different seasons by infrared thermography". Journal of Applied Animal Welfare Science 9 (2006): 211-221.

6. Tunley BV and Henson FMD. "Reliability and repeatability of thermographic examination and the normal thermographic image of the thoracolumbar region in the horse". Equine Veterinary Journal 36 (2004): 306-312.

7. MacPhee M and University of Prince Edward Island. Quantitative assessment of saddle fit using thermography. Undergraduate thesis (2008) University of Prince Edward Island, Charlottetown, P.E.I. (2008).

8. Dohoo., IR., et al. Veterinary Epidemiologic Research, $2^{\text {nd }}$ edition VER, Inc., Charlottetown, P.E.I., Canada (2009).

9. Venables WN and Ripley BD. Modern applied statistics with S-Plus, Third edition. Springer (2009).

10. Loughin CA and Marino DJ. "Evaluation of thermographic imaging of the limbs of healthy dogs". American Journal of Veterinary Research 68 (2007): 1064-1069.

11. Head MJ and Dyson S. "Taking the temperature of equine thermography”. Veterinary Journal 162 (2001): 166-167.
12. Zaproudina N., et al. "Reproducibility of infrared thermography measurements in healthy individuals". Physiological Measurement 29 (2008): 515-524.

13. Weil M., et al. "Diagnostic validity of thermography in equine lameness". Tierarztliche Praxis Ausgabe G, Grosstiere/Nutztiere (1988).

14. Turner TA. "Diagnostic thermography". Veterinary Clinics of North America Equine Practice 17 (2001): 95-113.

15. Autio E., et al. "Thermographic evaluation of the lower critical temperature in weanling horses". Journal of Applied Animal Welfare Science 10 (2007): 207-216.

16. Ousey JC., et al. "Thermoregulation and total body insulation in the neonatal foal". Journal of Thermal Biology 1 (1992): 1-10.

17. Hinchcliff KW., et al. "Equine exercise physiology: The science of exercise in the athletic horse". Elsevier Saunders, Edinburgh; New York (2008).

18. Levet T., et al. "Distal limb cast sores in horses: Risk factors and early detection using thermography". Equine Veterinary Journal 41.1 (2009): 18-23.

19. Palmer SE. "Use of the Portable Infrared Thermometer as a Means of Measuring Limb Surface Temperature in the Horse". American Journal of Veterinary Research 42 (1981): 105-108.

20. Palmer SE. "Effect of ambient temperature upon the surface temperature of the equine limb". American Journal of Veterinary Research 44 (1983).

21. Ivana Knıžková Kunc Ṕ., et al. "Evaluation of naturally ventilated dairy barn management by a thermographic method". Livestock Production Science 77 (2002): 349-353.

22. Morgan K. "Thermoneutral zone and critical temperatures of horses". Journal of Thermal Biology 23 (1998): 59-61.

23. Olowofela JA., et al. "Effect of environmental factors (relative humidity) on thermal signature of buried objects". New York Science Journal 4.3 (2011): 54-57.

\section{Assets from publication with us}

- Prompt Acknowledgement after receiving the article

- Thorough Double blinded peer review

- Rapid Publication

- Issue of Publication Certificate

- High visibility of your Published work

Website: www.actascientific.com/

Submit Article: www.actascientific.com/submission.php Email us: editor@actascientific.com

Contact us: +919182824667 